\title{
Cervical cancer incidence in young women: a historical and geographic controlled UK regional population study
}

\author{
A Patel', K Galaal', C Burnley², K Faulkner², P Martin-Hirsch ${ }^{3}$, MJ Bland ${ }^{4}$, S Leeson ${ }^{5}$, H Beer ${ }^{6}$, S Paranjothy $^{6,7}$, \\ P Sasieni ${ }^{8}$ and R Naik'
}

'Northern Gynaecological Oncology Centre, Queen Elizabeth Hospital, Sheriff Hill, Gateshead Tyne and Wear NE9 6SX, UK; ${ }^{2}$ The North East, Yorkshire and Humber Quality Assurance Reference Centre, Leeds, UK; ${ }^{3}$ Preston Royal Infirmary, Preston, UK; ${ }^{4}$ Department of Health Sciences, University of York, York, UK; ${ }^{5}$ Department of Obstetrics and Gynaecology, Betsi Cadwaladr University Health Board, North Wales, UK; ${ }^{6}$ Screening Division, Public Health Wales NHS Trust, Wales, UK; ${ }^{7}$ Department of Primary Care and Public Health, Cardiff University, Wales, UK; ${ }^{8}$ Centre for Cancer Prevention, Wolfson Institute of Preventive Medicine, Queen Mary University of London, London, UK

BACKGROUND: The commencing age of cervical screening in England was raised from 20 to 25 years in 2004. Cervical cancer incidence in young women of England is increasing. It is not clear if this is due to either greater exposure to population risk factors or reduced cervical screening.

METHODS: We measured if the relative risk of cervical cancer in younger women (20-29 years) of the north-east of England (NE) differed to that of women aged 30yrs and above since 2004. We also measured average annual percentage change (AAPC) in the 3 yr moving average incidence for all age-groups. Regional screening coverage rate and population risk factors were reviewed. Comparisons were made with Wales where screening continues to commence from the age of $20 \mathrm{yrs}$.

RESULTS: Cervical cancer incidence in women aged 20-29 increased annually by an average of 10.3\% between 2000 and 2009. The rise in women aged 30-39 was less steep (3.5\%/year) but no significant rise was observed in women aged 40-49. Socioeconomic factors remained stable or improved during the time period except for the incidence of chlamydia, herpes simplex and in particular, genital warts, which increased significantly in young women. Data from Wales show similar results.

CONCLUSION: The incidence of cervical cancers in young women of the NE is increasing. The rise in incidence is unrelated to the change in screening policy in 2004. Close monitoring of incidence in young women and a greater attempt to reverse the current decline in screening coverage of women aged 25-29 years are recommended.

British Journal of Cancer (2012) 106, 1753-1759. doi:10.1038/bjc.2012.148 www.bjcancer.com

Published online 24 April 2012

(c) 2012 Cancer Research UK

Keywords: cervical cancer; incidence; screening; cervical intra-epithelial neoplasia; human papilloma virus; sexually transmitted disease

Recently published analyses of trends in cervical cancer incidence in England suggest a rise in incidence in young women, particularly in the north-east of England (NE) (Foley et al, 2011; NCIN, 2011). Although wide variability in completeness of cancer registration (Swerdlow et al, 1993; Bullard et al, 2000; Bernays et al, 2002) limits interpretation, these results are alarming and of significant public health interest. Whether the rise in incidence is due to either greater exposure to risk factors or reduced cervical screening activity remains unclear.

The National Health Service Cervical Screening Programme (NHSCSP) has achieved remarkable success in the reduction of incidence of cervical cancer. An estimate of lives saved per year by screening in the United Kingdom varies between 1100 and 4500 according to the mathematical models used (Sasieni et al, 1996; Peto et al, 2004b). Despite such successes, commencing age of screening in England was raised from 20 to 25 years in 2004 (NHSCSP, 2003). This was in response to a study that concluded that the protection offered by negative cytology improves with age

*Correspondence: R Naik; E-mail: raj.naik@ghnt.nhs.uk

Received II November 2011; revised 21 March 2012; accepted 22 March 2012; published online 24 April 2012
(Sasieni et al, 2003). Following this policy change, the value and sensitivity of cervical screening in young women has been the subject of considerable debate. Although proponents have argued for the potential life years saved, opponents have raised concerns of physical and psychological harm to women and cost to society. In June 2009, an independent Advisory Committee for Cervical Screening in England reviewed the evidence and upheld the decision of cervical screening policy change of 2004 (ACCS, 2009). Analyses of an extended data set in 2009 also reported that screening of women aged 20-24 years had little or no impact on cancer incidence under the age of 30 years (Sasieni et al, 2009). In 2011, the cervical screening programme of Northern Ireland was amended with policy changes similar to England (NICSP, 2010). Scotland and Wales continue to offer the cervical screening from the age of 20 years.

The International Agency for Research on Cancer (IARC) in 1986 reported that the sensitivity of cervical cytology or the sojourn time period of a premalignant disease is not age dependent (IARC, 1986). More recent evidence from Wales (Rieck et al, 2006) and Sweden (Andrae et al, 2008) showed cytological screening to be effective in young women. Similar conclusions were reached by Peto et al (2004b) and Sigurdsson and Sigvaldason (2007) in their review of invasive and preinvasive cervical disease in England and 
Iceland, respectively. However, the relevance of these findings has been called into question (Sasieni et al, 2010).

The withdrawal of cervical screening from 20 to 24 years in England is the first occasion globally, where a population cancer screening programme has been cut back. Any subsequent increase in cancer incidence in young women will naturally be attributed to the policy change. However, the true and full effect can only be revealed by prospectively following the birth cohort (born in 1985 or after) directly affected by the change in policy. Such women will only turn 30 years in 2014 and cancer incidence data may not be available until 2017. Alternatively, a thorough review of multisource cancer registrations, screening coverage and population risk factors in the years immediately prior to and after the change in policy, particularly if compared with other nations of the United Kingdom, may clarify the causative factors responsible for the rise in cervical cancer incidence in young women.

In this study, we describe the trends in incidence of cervical cancer in young women within a well-defined geographical region of the NE between 2000 and 2009, and compare our results with that of Wales where women continued to be offered screening from the age of 20 years.

\section{MATERIALS AND METHODS}

All cervical cancer cases diagnosed between 2000 and 2009 within five predefined postcodes (NE, CA, DH, SR and TD) were identified from a regional cancer registry, cancer centres, cancer units and pathology laboratories of the NE and Cumbria. Duplicate cases were removed. Age at diagnosis, International Federation of Gynecology and Obstetrics (FIGO) stage, year of diagnosis and prior screening history were collected for each case. High-grade intra-epithelial neoplasia (HG-CIN, ICD10 code D06) registrations between 2000 and 2009 for the North-East Cancer Network $(\mathrm{NECN})$ were obtained from the regional cancer registry. Midyear population estimates were obtained from the Office of National Statistics (ONS).

We first confirmed if the relative risks for all and FIGO stage 1B or higher cases in three age groups $(20-24,25-29$ and 30 years and above) differed between two calendar periods, 2000-2004 and 2005-2009. We then assessed if relative risk in younger age groups (20-24 and 25-29 years) differed compared with 30 years and above between these two calendar periods. For this analysis we used Poisson regression, with number of cases as an outcome, adjusting for the populations' size as an exposure and using age group, calendar period and the interaction between age group and calendar period as predictors. A similar analysis was performed for HG-CIN registrations for the NECN.

We then measured an average annual percentage change (AAPC) in the 3-year moving average incidence rate for various age groups by fitting a regression line to the natural logarithm of the rates using calendar year as a regressor variable and allowing for significant trend changes between 2000 and 2009. The AAPC is a weighted average measure of all episodes of differing trend in cancer incidence over a fix time period. The weighted average incorporates an annual percentage change (APC) in incidence, the slope coefficients of regression trend line and the duration (in years) of each episode (NCI, 2011a). This analysis was performed for all, FIGO stage 1B or higher, FIGO stage 1A cancer cases and HG-CIN cases. This enabled us to measure if the rise in risk was steady or if there were any significant point changes in the trend of cervical cancer incidence.

As women born prior to 1984 would have been invited for screening from the age of 20 years, and those born in 1985 or after would not have been invited until age 25 years (in 2009), it is then of interest, that we compare cervical cancer incidence at ages 20-24 years in women invited from the age of 20 years with those not invited until the age of 25 years keeping an equal number at different ages. Similar analyses for women aged 25-29 years would not be possible for a further few years as the cohort entered screening in 2009.

To determine route to diagnosis of cervical cancer (symptom $v s$ screen detected) and screen category, we reviewed cervical screening histories of women aged 20-29 years. Comparisons were made between the two calendar periods. The screening coverage rates for the NE were obtained from The North East, Yorkshire and Humber Quality Assurance Reference Centre (NEYHQARC). The regional and national population trends on various risk factors for cervical cancer (e.g., socioeconomic factors, smoking, sexually transmitted diseases, teenage conceptions and terminations, and migration) during the 10 -year study period were analysed. Data for these factors were obtained from the ONS, Health Protection Agency and the Department of Health.

A quality assured registration of cervical cancer cases along with HG-CIN registration, population statistics, screening coverage rates and trends in various risk factors for the Wales population were obtained from the Welsh Cancer Intelligence and Surveillance Unit, ONS, Cervical Screening Wales and Public Health Wales NHS Trust, respectively. Similar statistical analyses and comparisons were made with the NE data.

All statistical analyses were two-sided. $P$-value $<0.05$ was considered significant. Statistical analyses were performed using Stata 10 (Stata Corp., College Station, TX, USA) except for the AAPC analysis that was performed using the 'JoinPoint' software from the Surveillance Research Program of the US National Cancer Institute (NCI, 2011b).

\section{RESULTS}

A total of 1061 cervical cancer cases were diagnosed between 2000 and 2009 in the defined geographical region of the NE: 512 cases between the years 2000 and 2004, and 549 cases between 2005 and 2009 with overall incidences of 8.4 and 8.9 per 100000 womenyears, respectively. There were $152(14 \%)$ cases in women aged between 20-29 years between 2000 and 2009, of which $39(26 \%)$ were aged $20-24$ years. Overall, 54 out of 940 cases $(5.7 \%)$ of cervical cancers were not registered with the regional cancer registry. These missing cases showed no obvious trends in age, FIGO stage or geographical distribution (Supplementary Table S1). In addition, $14 \%$ of cases registered at regional cancer registry either had missing or erroneous FIGO stage.

Table 1 and Supplementary Figure S1 show the Poisson regression analysis and differences between the two calendar periods (2000-2004 and 2005-2009) in cervical cancer and HG-CIN incidence in the NE. There is strong evidence for all cancers $\left({ }^{*} P=0.001\right)$ and Stage $1 \mathrm{~B}$ and higher cancers $\left({ }^{\star} P=0.005\right)$ that the relative risks among the three age groups differed between two time periods. The interaction also gave sufficient evidence $\left({ }^{\star} P=0.04\right)$ for women aged $20-24$ years, and strong evidence $\left({ }^{*} P=0.004\right)$ for women aged $25-29$ years that their relative risk has increased compared with women aged 30 years and above since 2004. Similar analyses limited to FIGO Stage 1B and higher returned significance values of 0.068 and 0.021 for women aged 20-24 and 25-29 years, respectively. When Poisson regression was performed with four age groups (20-24, 25-29, 30-34 and 35 years and above) we found similar results for 20-24 and 25-29 age groups (Table 1b). In addition, we found that the relative risk was higher in the 30-34 years age group since 2004 compared with 35 years and above. However, when the analysis was performed excluding cases from 2009 (data not shown) to eliminate the effect of media coverage following the diagnosis and death of a UK celebrity from cervical cancer (Jade Goody effect), the relative risk remained higher for the 20-24 and 25-29 age groups, but not for women aged 30-34 years. Analysis of HG-CIN, expectedly, showed significant reduction in incidence in the 20-24 years age group since the withdrawal of screening 
Table I Age group-specific incidence of cervical cancer and HG-CIN, and Poisson regression of interaction between calendar period and age groups in the north-east of England and Wales



Abbreviations: $\mathrm{Cl}=$ confidence interval; FIGO = International Federation of Gynecology and Obstetrics; HG-CIN = high-grade intra-epithelial neoplasia; IRR = incidence rate ratio; Ref. = reference age group. ${ }^{a}$ Incidence rate per 100000 women calculated using age group-specific incidence for each year of the study period. ${ }^{b}$ IRR and Poisson regression to determine whether the relative risk of age groups has differed between two time periods. ${ }^{\mathrm{I} R R}$ and Poisson regression to determine whether the relative risk of younger age groups differed between two time periods compared with the $30+$ or $35+$ years age group (Table Ia and Ib, respectively). ${ }^{\mathrm{d}} \mathrm{HG}-\mathrm{CIN}$ incidence data for NE available up to 2008

(difference of 201 per 100000$)$. However, an equivalent and considerable rise in incidence (difference of 131 per 100000 ) was observed in the 25-29 years age group.

Table 2 presents an AAPC in 3-year moving average incidence of cervical cancer and HG-CIN to assess whether the change in incidence was steady over time in the different age groups of the NE. The rise in AAPC for age groups 20-24 and 25-29 years was steady, similar and significant from public health perspective (Supplementary Figure S2). Combining these two groups to 20-29 years (and adjusting for age group), the AAPC in all stages, stage $1 \mathrm{~B}$ and higher and stage $1 \mathrm{~A}$ cervical cancer were $10.3 \%$ (95\% CI: 4.4 to $18.7 \%), 15.2 \%$ (12.0 to $18.5 \%$ ) and $5.3 \%$ ( -3.7 to $15.2 \%)$, respectively. With respect to all cervical cancer cases, the rise in AAPC decreased with age from 30 to 39 years and the rates changed little over time in those aged 40 years and above. When analyses were performed excluding cases from 2009 (data not shown), eliminating the effect of media coverage (Jade Goody effect) and any resulting increase in screening, we obtained similar results except that between 2000 and 2008 there was virtually no increase in cancer rates in those aged 30-39 years. Average annual percentage change analysis of HG-CIN incidence suggests a gradual reduction in HG-CIN cases in 20-24 years and a rise in 25-29 years age group.

A birth cohort analysis of women aged 20-24 years, comparing those invited from the age of 20 yrs (born before 1985) with those not invited until age of 25 years (born after 1984), revealed 6 cases (2.6 per 100000 women) against 11 cases (4.4 per 100000 women), respectively $(P=0.399)$. Analyses limited to stage $1 \mathrm{~B}$ and higher cases showed that there were three cases (1.3 per
$100000)$ in those invited from the age of 20 years compared with five (2.0 per 100000$)$ in those not invited until age of 25 years $(P=0.794)$.

Supplementary Table S2 shows an analysis of screening histories of young women (20-29) in the NE diagnosed with cervical cancer. More women aged 20-24 years presented with symptoms in 2005-2009 compared with 2000-2004 $\left({ }^{*} P=0.048\right)$. The cumulative incidence per 100000 women of FIGO stage $1 \mathrm{~B}$ and higher cases in this age group was 1.2 and 3.1 during 2000-2004 and 2005-2009, respectively. We did not find any significant differences in screen categories between the two calendar periods for either the 20-24 years or the 25-29 years age groups. In addition, there was no significant difference in presentation for the 25-29 years age group between the two calendar periods. However, significantly less women aged 25-29 years in the 2005-2009 had two previous negative smears $\left({ }^{\star} P=0.042\right)$.

Supplementary Figure S3 shows cervical screening coverage rates for women aged 20-24 and 25-29 years in the NE and Wales. As coverage is defined as having been screened in the previous 5 years, it is inevitable that coverage of 25 year olds will decrease if women aged 20-24 years are no longer invited for screening. Screening coverage in Wales appears to follow similar trends except that the screening coverage for women aged 20-24 years did not show a dramatic decrease since 2004 as Wales continue to screen this age group. Coverage rates were comparatively higher in the NE than in Wales for women aged 20-24 years until 2004 and 25-29 years until 2008.

Figure 1 shows trends in various population risk factors in the $\mathrm{NE}$ and Wales that may have had potential effects on cervical 
Table 2 Average annual percentage change (AAPC) in 3-year moving average incidence per 100000 women of cervical cancer and HG-CIN in the north-east of England and Wales

\begin{tabular}{|c|c|c|c|c|c|c|c|}
\hline $\begin{array}{l}\text { Cancer and } \\
\text { HG-CIN cases }\end{array}$ & $\begin{array}{c}\text { Age groups } \\
\text { (years) }\end{array}$ & \multicolumn{3}{|c|}{ North-east of England } & \multicolumn{3}{|c|}{ Wales } \\
\hline All stages & $\begin{array}{l}20-24 \\
25-29 \\
20-29 \\
30-39 \\
40-49 \\
50-64 \\
65+\end{array}$ & $\begin{array}{r}3.4 \\
9.5 \\
6.5 \\
17.1 \\
9.6 \\
8.8 \\
10.4\end{array}$ & $\begin{array}{r}7.4 \\
21.0 \\
13.7 \\
22.2 \\
9.0 \\
6.4 \\
8.3\end{array}$ & $\begin{array}{l}\text { I2.8 (6.0 to } 20.1) \\
\mid 1.3(4.4 \text { to | } 8.7) \\
10.3(6.7 \text { to | } 4.0) \\
3.5(0.8 \text { to } 6.2) \\
2.7(-2.9 \text { to } 8.6) \\
-4.3(-5.9 \text { to }-2.6) \\
\quad 1.2(-0.3 \text { to } 2.8)\end{array}$ & $\begin{array}{r}3.5 \\
10.3 \\
6.9 \\
18.8 \\
17.5 \\
12.5 \\
16.1\end{array}$ & $\begin{array}{r}5.3 \\
20.9 \\
12.5 \\
22.0 \\
18.0 \\
12.3 \\
15.3\end{array}$ & $\begin{array}{r}\text { II.4 (0.4 to } 23.5) \\
\text { I2.4 (9.8 to I5.0) } \\
\text { II.8 (8.| to I5.6) } \\
\text { I.5 (-2.9 to } 6.2) \\
0.3(-2.4 \text { to } 3.2) \\
-0.5(-2.8 \text { to } 1.9) \\
-1.3(-2.7 \text { to } 0.1)\end{array}$ \\
\hline $\mathrm{FIGO}$ IB + & $\begin{array}{l}20-24 \\
25-29 \\
20-29 \\
30-39 \\
40-49 \\
50-64 \\
65+\end{array}$ & $\begin{array}{r}1.4 \\
3.8 \\
2.6 \\
9.9 \\
6.2 \\
7.6 \\
10.3\end{array}$ & $\begin{array}{r}3.7 \\
12.6 \\
7.9 \\
12.2 \\
7.9 \\
5.7 \\
8.3\end{array}$ & $\begin{array}{l}20.8(6.8 \text { to } 36.5) \\
13.6(7.0 \text { to } 20.6) \\
15.2(12.0 \text { to } 18.5) \\
1.3(-1.6 \text { to } 4.2) \\
5.4(2.1-8.9) \\
-3.5(-6.0 \text { to }-1.0) \\
-2.8(-5.5 \text { to } 0.0)\end{array}$ & & & \\
\hline FIGO I A & $\begin{array}{l}20-24 \\
25-29 \\
20-29 \\
30-39 \\
40-49 \\
50-64 \\
65+\end{array}$ & $\begin{array}{l}1.9 \\
5.7 \\
3.8 \\
7.1 \\
3.4 \\
1.2 \\
0.1\end{array}$ & $\begin{array}{r}3.7 \\
8.4 \\
5.9 \\
10.0 \\
1.1 \\
0.7 \\
0.0\end{array}$ & $\begin{array}{r}6.8(-0.5 \text { to } 14.6) \\
5.2(-4.1 \text { to } 15.4) \\
5.3(-3.7 \text { to } 15.2) \\
6.5(1.0 \text { to } 12.3) \\
-4.5(-16.5 \text { to } 9.3) \\
-8.9(-19.9 \text { to } 3.6) \\
-18.6(-36.9 \text { to } 5.1)\end{array}$ & & & \\
\hline & & $2000-02$ & $2006-08^{b}$ & $\operatorname{AAPC}(95 \% \mathrm{Cl})$ & $2000-02$ & 2007-09 & $\operatorname{AAPC}(95 \% \mathrm{Cl})$ \\
\hline HG-CIN & $\begin{array}{l}20-24 \\
25-29 \\
30-39 \\
40-49 \\
50-64\end{array}$ & $\begin{array}{r}453.7 \\
483.8 \\
288.1 \\
91.0 \\
16.4\end{array}$ & $\begin{array}{r}203.1 \\
611.9 \\
298.7 \\
83.7 \\
10.5\end{array}$ & $\begin{array}{l}-12.2(-14.0 \text { to }-10.3) \\
4.3(3.1 \text { to } 5.5) \\
0.9(-0.2 \text { to } 2.0) \\
-0.8(-2.6 \text { to } 1.0) \\
-7.2(-9.3 \text { to }-5.1)\end{array}$ & $\begin{array}{r}205.9 \\
293.9 \\
175.4 \\
52.7 \\
17.8\end{array}$ & $\begin{array}{r}363.8 \\
511.7 \\
253.4 \\
48.9 \\
23.9\end{array}$ & $\begin{array}{l}8.4(6.3 \text { to } 10.6) \\
8.8(6.6 \text { to } 10.9) \\
5.3(3.5 \text { to } 7.1) \\
6.7(0.1 \text { to } 13.8) \\
3.9(-1.1 \text { to } 9.2)\end{array}$ \\
\hline
\end{tabular}

Abbreviation: $\mathrm{Cl}=$ confidence interval. ${ }^{\mathrm{T}}$ Three-year average incidence rate per 100000 women. ${ }^{\mathrm{b}}$ Incidence data available up to 2008.

cancer incidence rates during the 10 -year study period. Incidence of the more common sexually transmitted infections increased substantially in the NE and Wales (Figures 1A-D): Numbers of episodes of Chlamydia and Herpes nearly doubled in both the 20-24 and the 25-34 years age groups in the NE between 2000 and 2009 , with a significant rise in Wales as well. The number of new episodes of genital warts in the NE also increased by almost $25 \%$ in the 25-34 years age group during the last 10 years. There were no major changes observed in the other population risk factors during the study period.

Poisson regression, AAPC analyses and trends in the incidence of cervical cancer and HG-CIN incidence in Wales, where screening continued from the age of 20 years, are detailed in Tables 1 and 2 and Supplementary Figure S4. Poisson regression and AAPC analyses revealed that the change in incidence of cervical cancer in young women of Wales since 2004 is significant, steady and similar to the NE. While registration of HG-CIN in 20-24 years has dramatically and expectedly decreased in the NE, a significant increase is observed in Wales. A rise in HG-CIN registrations in the 25-29 years age group is also substantial and similar to the NE.

\section{DISCUSSION}

We identified a significant increase in the incidence of cervical cancer in young women in the NE since the withdrawal of screening from the $20-24$ years age group in 2004. Although this is predominantly in women aged 25-29 years, there is also evidence of an increase in women aged 20-24 years. It has been argued that the inclusion of stage $1 \mathrm{~A}$ cervical cancer cases in the incidence analyses is likely to overestimate the potential harm of withdrawal of screening (Sasieni et al, 2010). We therefore analysed stage IB and higher cancers separately in the NE and still observed a significant increase in incidence in women aged 25-29 years and a trend towards significance in women aged 20-24 years since 2004.

This does not appear to be a spurious rise in incidence due to increased screening activity (media coverage/'Jade Goody' effect), higher registration rates or improved detection after the introduction of liquid-based cytology (LBC). The significant test results after excluding cases from 2009 rules out the effects of media coverage. Unregistered cases at the regional cancer registry were comparatively high during 2005-2007, precluding the possibility that the increased incidence may be related to improved registrations. While a meta-analysis of LBC showed up to $12 \%$ better sensitivity (NICE, 2003), transition from conventional cervical cytology to LBC processing across the United Kingdom was completed in 2008 (NHSCSP, 2008) and most laboratories in the NE completed the transition by 2006 . However, this could not explain the rise in incidence as this would otherwise have affected women of all ages. Also, if related to LBC, after an initial rise there would be an expectant decline in rate of detection and the incidence rate would decrease to baseline levels. 
A

STI rates 20-24, NE and Wales

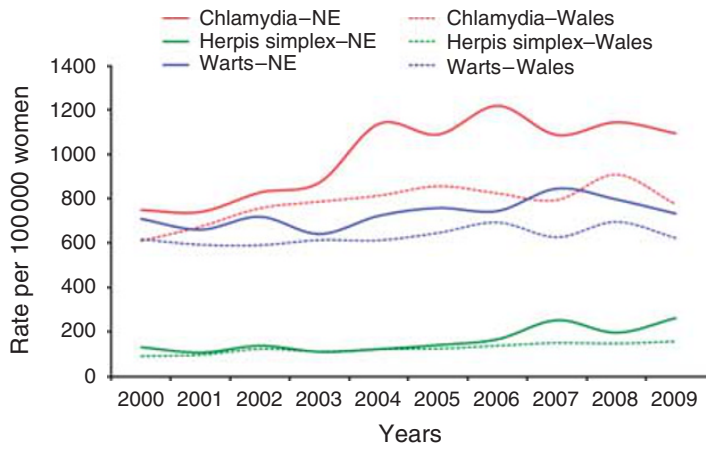

C

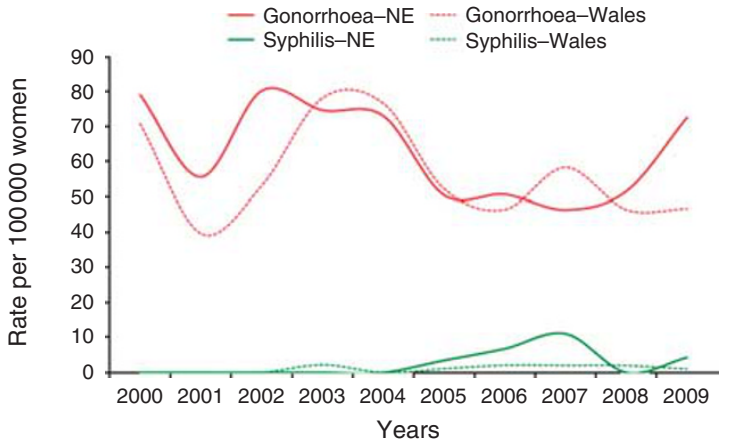

$\mathbf{E}$

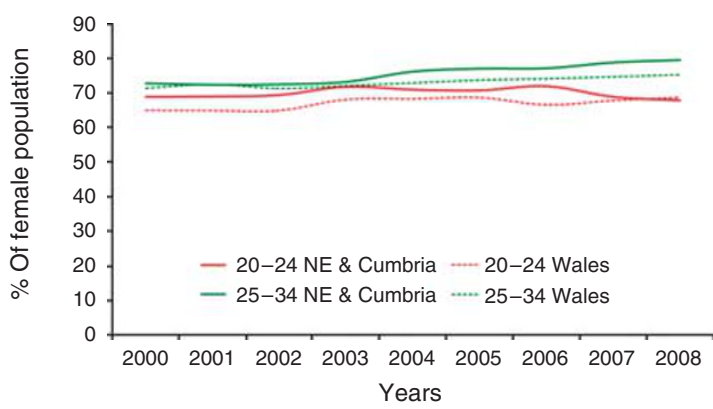

G Teenage conceptions and terminations

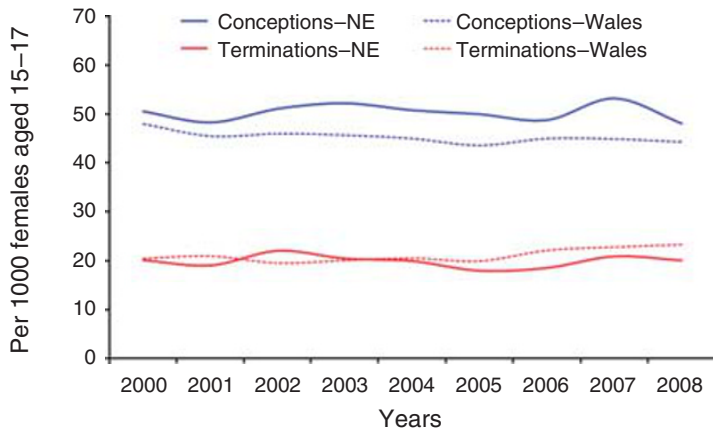

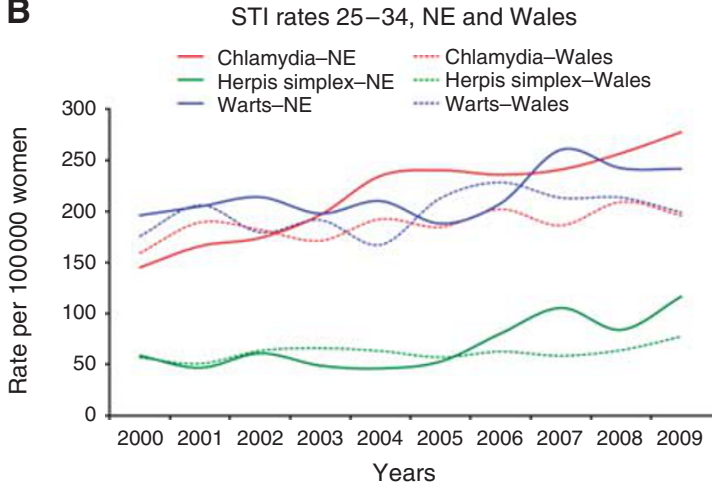

D STI rates 25-34, NE and Wales

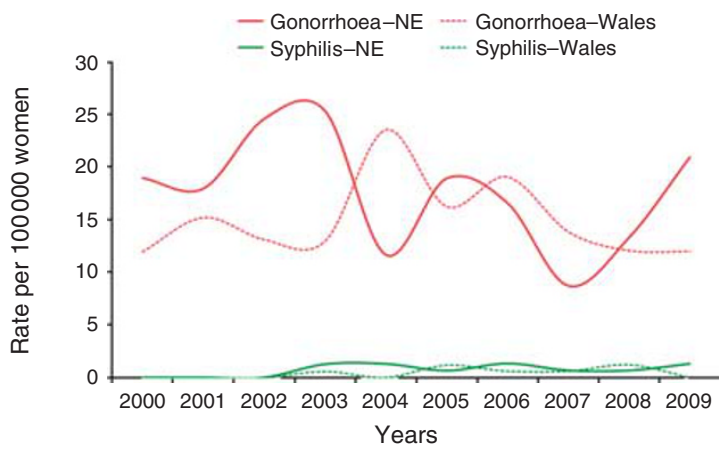

F Cumulative migration (all ages and both sex)

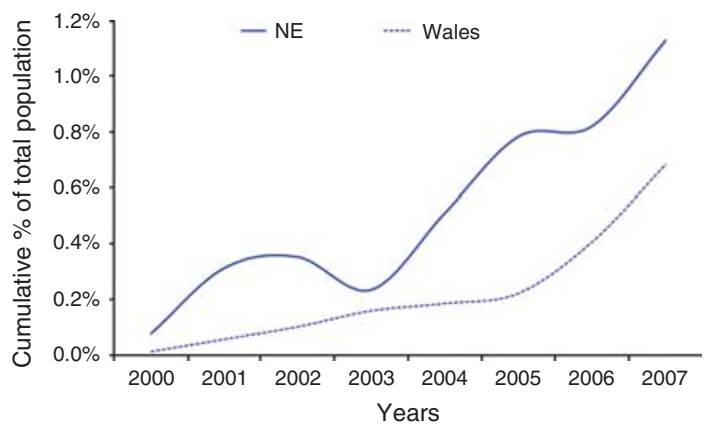

H Smoking

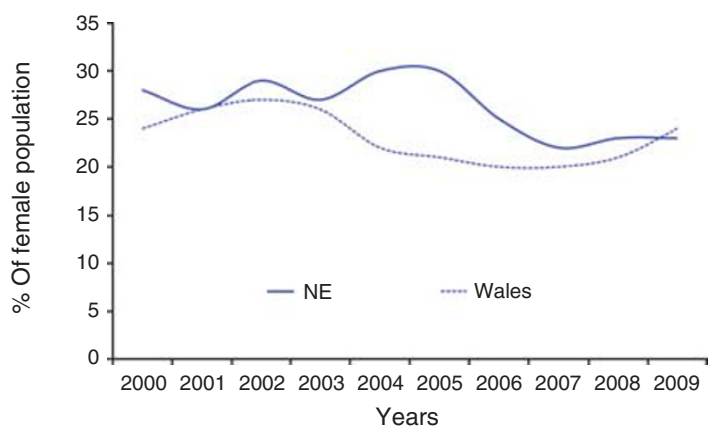

Figure I Trends in population risk factors for cervical cancer in NE and Wales.

If the rise in incidence is real, it is likely to be due to either reduced screening activity (including the 2004 policy change in England) or a greater risk through greater exposure to associated or predisposing factors or both. This necessitated examination of changes in the screening coverage and risk factors. Screening coverage has decreased dramatically in women aged 20-24 years since 2004 due to the direct effect of withdrawal of screening in this group. Screening coverage of women aged 25-29 years has decreased as well albeit gradually over the last 10 years, which is consistent with national and international trends in this age group (Lancucki et al, 2010). On the assumption that a substantial proportion of HG-CIN does not regress, it is inevitable that a reduced detection and treatment of HG-CIN in women aged 20-24 years of age following withdrawal of screening will result in a 
greater detection of cases with HG-CIN and possibly cancer after the age of 25 years simply through a delay in identification. This is unlikely to be the explanation for a rise in the incidence of cancer in the 25-29 years age group, as women aged 25 years in 2008 would have been offered screening from age 20 years.

The average annual percentage increase in incidence rates of cancer in young women of NE aged 20-29 years is identical at 10.3\%/year during 2000-2004 and 2005-2009. This supports a gradual steady rise rather than a point change in incidence such as withdrawal of screening in 2004. Additionally, the birth cohort analysis of 20-24 years age group (born before and after 1 January 1985) does not support any deleterious effects of withdrawal of screening in this age group, although the number of cases are small and it may be too early to infer.

Investigation of prior screening history of cases diagnosed with cervical cancer aged 25-29 years in the NE showed no difference in clinical presentation or screen category. It did, however, show fewer women in 2005-2009 with a prior history of two negative smears. This represents reduced screening activity in young women and therefore could explain the rise in incidence. However, in the 25-29 years age category prior to the policy change in 2004, $62 \%$ of cases had a history of two (or more) previous negative smears, which supports the concept that a negative smear in this group of women is less protective in the following 3-6 years than in the older women (Sasieni et al, 2003).

Analyses of population risk factors revealed a substantial rise in the incidence of chlamydia, herpes simplex and genital warts since 2000 , particularly in younger women in the NE. It may seem logical then to assign the increased risk of cervical cancer to an increased HPV prevalence and/or change in sexual behaviour in recent years. There is, however, no direct evidence to support any significant change in sexual behaviour or an explosive rise in HPV prevalence in such a short duration. In fact, studies of the prevalence of high-risk HPV in the NW of England suggest a modest gradual rise over the last two decades (Peto et al, 2004a; Sargent et al, 2008).

Interestingly, the incidence of cervical cancer in young women in Wales aged 20-29 years shows very similar trends to that of the NE, despite continued screening of women aged 20-24 years. Trends in the population risk factors including sexually transmitted diseases in young women of Wales are similar to the NE as well. Additionally, while a rise in HG-CIN incidence in women of Wales aged 25-29 years is very similar to the NE, incidence in women aged 20-24 years has risen significantly despite continued screening in this age group. This supports a rise in the background risk rather than reduced screening activity as the cause for an increased incidence of cervical cancer in young women.

In summary, this study confirms the steady rise in incidence of cervical cancer in young women as observed by Foley et al (2011). In addition, a thorough review of regional cervical cancer cases with regional screening coverage, population risk factors, HG-CIN incidence and comparison with the Wales data suggests that the rise in incidence in young women is unrelated to the change in policy of withdrawal of screening from women aged 20-24 years, and is associated with an increase in exposure to background risk factors including HPV. The birth cohort of young women greatest affected by the 2004 change in policy will have recently entered the screening programme having just turned 25 years. The direct and indirect effects of HPV vaccination programme on cervical cancer incidence will not be genuinely evident until the vaccinated cohort has reached the age of 35 years, the peak incidence age for cervical cancer (Bauch et al, 2010; Harper et al, 2010). The issue of cervical screening in young women therefore continues to be relevant for the foreseeable future. While STRATEGIC trial is underway to explore various strategies to improve the uptake of cervical screening in young women (Kitchener, 2011), we recommend close surveillance of incidence in younger women over the coming years and a greater attempt to reverse the current decline in screening coverage for women aged 25-29 years.

\section{ACKNOWLEDGEMENTS}

Our sincere thanks and acknowledgement goes to the following individuals and organisations that has helped and contributed to the study by providing relevant data without which this study would not have been possible. Helen Jaretzke, Data manager, NGOC, Gateshead; Martin Jones, Pathology Dept, QE Hospital, Gateshead; The Northern and Yorkshire Cancer Registry and Information Service (NYCRIS), Leeds; The North East Yorkshire and the Humber Quality Assurance Reference Centre (NEYHQARC), Leeds; Cancer Care Alliance (CCA), Middlesbrough; The Office of National Statistics (ONS), London; Nomis (Labour and Market Statistics), Durham; The Health Protection Agency (HPA), London; Welsh Cancer Intelligence and Surveillance Unit, Cardiff; Cervical Screening Wales, Cardiff; Public Health Wales NHS Trust, Cardiff.

\section{Conflict of interest}

All authors have declared conflict of interest statements. (1) PS receives salary from institutional grant from CRUK, although no remuneration was directly paid for the submitted work. (2) RN is involved in an expert advisory group to sanofi pasteur, sponsored presentation for Baxter Ltd and receives royalties for being coauthor of Bonney's gynaecological surgery. (3) None of the author's spouses, partners or children has any financial relationships that may be relevant to the submitted work. (4) None of the authors have non-financial interests that may be relevant to the submitted work.

\section{Author contributions}

RN conceived the study. AP collected data and performed analyses. CB provided screening coverage data for the NE. SL, HB and SP provided data for Wales. KG and RN contributed to the analysis of data. MJB and PS provided statistical and epidemiological support for the analysis. AP wrote first draft of the manuscript. RN, KG, $\mathrm{CB}, \mathrm{KF}, \mathrm{PM}-\mathrm{H}, \mathrm{SL}, \mathrm{HB}, \mathrm{SP}$ and PS revised the manuscript and contributed to the discussion. All authors critically appraised and approved the final manuscript.

Supplementary information accompanies the paper on British Journal of Cancer website (http://www.nature.com/bjc)

\section{REFERENCES}

ACCS (2009) Re-examination of current policy on cervical screening for women aged 20-24 years. www.dh.gov.uk/prod_consum_dh/groups/ dh_digitalassets/documents/digitalasset/dh_101366.pdf

Andrae B, Kemetli L, Sparen P, Silfverdal L, Strander B, Ryd W, Dillner J, Tornberg S (2008) Screening-preventable cervical cancer risks: evidence from a nationwide audit in Sweden. J Natl Cancer Inst 100(9): 622-629
Bauch CT, Li M, Chapman G, Galvani AP (2010) Adherence to cervical screening in the era of human papillomavirus vaccination: how low is too low? Lancet Infect Dis 10(2): 133-137

Bernays RL, Kollias SS, Khan N, Brandner S, Meier S, Yonekawa Y (2002) Histological yield, complications, and technological considerations in 114 consecutive frameless stereotactic biopsy procedures aided by open intraoperative magnetic resonance imaging. J Neurosurg 97(2): 354-362 
Bullard J, Coleman MP, Robinson D, Lutz JM, Bell J, Peto J (2000) Completeness of cancer registration: a new method for routine use Br J Cancer 82(5): 1111-1116

Foley G, Alston R, Geraci M, Brabin L, Kitchener H, Birch J (2011) Increasing rates of cervical cancer in young women in England: an analysis of national data 1982-2006. Br J Cancer 105(1): 177-184

Harper DM, Nieminen P, Paavonen J, Lehtinen M (2010) Cervical cancer incidence can increase despite HPV vaccination. Lancet Infect Dis 10(9): 594-595; author reply 595

IARC (1986) Screening for squamous cervical cancer: duration of low risk after negative results of cervical cytology and its implication for screening policies. IARC Working Group on Cervical Cancer Screening. Br Med J (Clin Res Ed) 293(6548): 659-664

Kitchener H (2011) STRATEGIes to increase Cervical screening uptake at first invitation (STRATEGIC): a cluster randomised trial. International Standard Randomised Controlled Trial Number (ISRCTN) Register, ISRCTN52303479. http://www.controlled-trials.com/ISRCTN 52303479/

Lancucki L, Fender M, Koukari A, Lynge E, Mai V, Mancini E, Onysko J, Ronco G, Tornberg S, Vessey M, Patnick J (2010) A fall-off in cervical screening coverage of younger women in developed countries. $\mathrm{J} \mathrm{Med}$ Screen 17(2): 91-96

NCI (2011a) Average Annual Percent Change (AAPC), Statistical Methodology and Applications Branch, National Cancer Institute. http:// surveillance.cancer.gov/joinpoint/aapc.html

NCI (2011b) JoinPoint Regression Software. Statistical Methodology and Applications Branch, National Cancer Institute. http://surveillance. cancer.gov/joinpoint/

NCIN (2011) Profile of Cervical Cancer in England: Incidence, Mortality and Survival, National Cancer Intelligence Network. http://www.ncin.org. uk/publications/reports/default.aspx\#type_specific

NHSCSP (2003) Modernising the NHSCSP: introduction of LBC and change in national policy. National Health Service Cervical Screening Programme (NHSCSP). http://www.cancerscreening.nhs.uk/cervical/news/009.html

NHSCSP (2008) NHS Cervical Screening Programme Annual Review 2008 (England) http://www.cancerscreening.nhs.uk/cervical/publications/ 2008review.html

NICE (2003) Technology Appraisal Guidance (TA69): Guidance on the use of liquid-based cytology for cervical screening: The National
Institute for Health and Clinical Excellence (NICE), http://guidance.nice. org.uk/TA69

NICSP (2010) NI Cancer Screening Programmes: Changes to the cervical screening policy. http://www.cancerscreening.hscni.net/cervical/08-HCERVICALWHATSNEW.html\#P7_233

Peto J, Gilham C, Deacon J, Taylor C, Evans C, Binns W, Haywood M, Elanko N, Coleman D, Yule R, Desai M (2004a) Cervical HPV infection and neoplasia in a large population-based prospective study: the Manchester cohort. Br J Cancer 91(5): 942-953

Peto J, Gilham C, Fletcher O, Matthews FE (2004b) The cervical cancer epidemic that screening has prevented in the UK. Lancet 364(9430): 249-256

Rieck GC, Tristram A, Hauke A, Fielder H, Fiander AN (2006) Cervical screening in 20-24-year olds. J Med Screen 13(2): 64-71; discussion 62-3

Sargent A, Bailey A, Almonte M, Turner A, Thomson C, Peto J, Desai M, Mather J, Moss S, Roberts C, Kitchener HC (2008) Prevalence of type-specific HPV infection by age and grade of cervical cytology: data from the ARTISTIC trial. Br J Cancer 98(10): 1704-1709

Sasieni P, Adams J, Cuzick J (2003) Benefit of cervical screening at different ages: evidence from the UK audit of screening histories. $\mathrm{Br} \mathrm{J}$ Cancer 89(1): 88-93

Sasieni P, Castanon A, Cuzick J (2009) Effectiveness of cervical screening with age: population based case-control study of prospectively recorded data. $\mathrm{Br}$ Med J 339: b2968

Sasieni P, Castanon A, Cuzick J (2010) The impact of cervical screening on young women: A critical review of the literature. In NHSCSP Publication 31. http://www.cancerscreening.nhs.uk/cervical/publications/nhscsp31.html

Sasieni PD, Cuzick J, Lynch-Farmery E (1996) Estimating the efficacy of screening by auditing smear histories of women with and without cervical cancer. The National Co-ordinating Network for Cervical Screening Working Group. Br J Cancer 73(8): 1001-1005

Sigurdsson K, Sigvaldason H (2007) Is it rational to start population-based cervical cancer screening at or soon after age 20? Analysis of time trends in preinvasive and invasive diseases. Eur J Cancer 43(4): 769-774

Swerdlow AJ, Douglas AJ, Vaughan Hudson G, Vaughan Hudson B (1993) Completeness of cancer registration in England and Wales: an assessment based on 2,145 patients with Hodgkin's disease independently registered by the British National Lymphoma Investigation. Br J Cancer 67(2): 326-329

This work is published under the standard license to publish agreement. After 12 months the work will become freely available and the license terms will switch to a Creative Commons Attribution-NonCommercial-Share Alike 3.0 Unported License. 